\title{
All-optical Unicast/Multicast Routing in WDM Networks
}

\author{
Javier E. Sierra*, Luis F. Caro ${ }^{\dagger}$, Fernando Solano ${ }^{\ddagger}$, Jose L. Marzo ${ }^{\dagger}$, Ramon Fabregat ${ }^{\dagger}$ and Yezid Donoso $^{\S}$ \\ ${ }^{*}$ GIDATI Research Group, Universidad Pontificia Bolivariana, Medellin, Colombia \\ Email: javier.sierra@upb.edu.co \\ ${ }^{\dagger}$ Institute of Informatics and Applications, University of Girona, Girona, Spain \\ ${ }_{\ddagger}^{\ddagger}$ Institute of Telecommunications, Warsaw University of Technology, Warsaw, Poland \\ ${ }^{\S}$ Computer Science Department, Universidad de los Andes, Bogota, Colombia
}

\begin{abstract}
Optical Transport Networks (OTN) must be prepared in terms of better resource utilization, for accommodating unicast and multicast traffic together. Light-trees have been proposed for supporting multicast connections in OTN. Nevertheless when Traffic Grooming is applied in light-trees, resources can be underutilized as traffic can be routed to undesirable destinations in order to avoid Optical-Electrical-Optical (OEO) conversions. In this paper, a novel architecture named $S / G$ Lighttree for supporting unicast/multicast connections is proposed. The architecture allows traffic dropping and aggregation in different wavelengths without performing OEO conversions. A heuristic that routes traffic demands using less wavelengths by taking advantage of the proposed architecture is designed as well. Simulation results show that the architecture can minimize the number of used wavelengths and OEO conversions when compared to light-trees.
\end{abstract}

\section{INTRODUCTION}

Wavelength Division Multiplexing (WDM) has allowed the fiber transmission capacity to greatly increase by supporting aggregate throughputs in the order of terabytes per second in just a single fiber. Nevertheless due to its great capacity, allOptical Transport Networks (OTN) can suffer from resource underutilization as not all connection request use the full capacity of a wavelength. In order to efficiently allocate the capacity of each wavelength channel (lightpath), several independent lower speed traffic [subwavelength connections, e.g., $O C-3(155 \mathrm{Mb} / \mathrm{s}), O C-12(622 \mathrm{Mb} / \mathrm{s})]$ must be multiplexed onto a single lightpath [e.g., $O C-48(2.5 \mathrm{~Gb} / \mathrm{s}), O C-192$ $(10 \mathrm{~Gb} / \mathrm{s})]$. The process of combining low-rate traffic onto high-capacity optical channels (lightpaths) is known in the literature as Traffic Grooming [1]. Traffic Grooming has been applied in order to improve the network resource utilization and operational cost.

There is a global increase in the demand of new applications that can take advantage of a multicast enabled network [2], [3]. In order to gain the advantages of deploying these applications in a multicast OTN, several architectures that consider both optical and electrical switching have been developed. Lighttrees [2], [4], [5] which is one of them, allows to fully deploy multicast traffic in a completely optical environment, a lighttree can be defined as an optical connection between one source to several destinations. The light-tree is created by using optical splitters that replicate one optical signal into

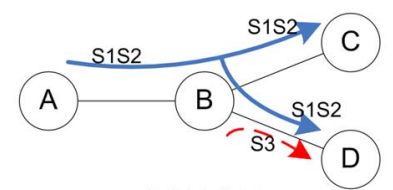

(a) Light-tree

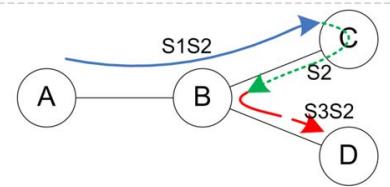

(c) Light-Trails

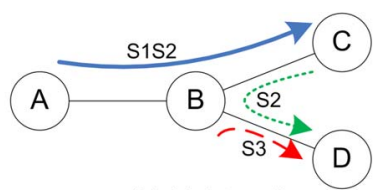

(b) Light-path

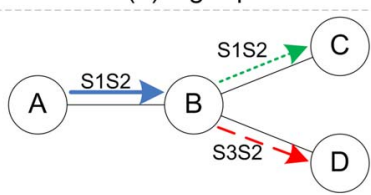

(d) Link-by-link

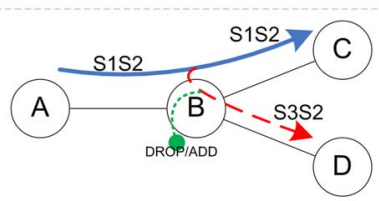

(e) S/G Light-tree

Fig. 1. Example, $S 1:\{A\} \rightarrow\{C\}, S 2:\{A\} \rightarrow\{C, D\}, S 1:\{B\} \rightarrow$ $\{D\}$; methods used for routing: a) Light-tree, b) Lightpath, c) Light-trails, d) Link-by-link, and e) $S / G$ Light-tree

several ones completely optical.

Traffic grooming allows to improve resource utilization for both unicast and multicast traffic. It consists of multiplexing traffic (usually statistically) from different demands in the same lightpath or light-tree, as well as to route one demand through several lightpaths and/or light-trees [6]. The routing of unicast and multicast traffic together in a network represents a challenge if we take into account the limitations of the WDM technology. Traditionally, there are two schemes for performing this. In the first scheme, light-trees groom together unicast with multicast traffic; thus OEO conversions are reduced but, unicast traffic can arrive to undesired nodes (incurring in bandwidth wastage). In the second scheme, unicast traffic is routed using lightpaths through different wavelengths than those used by light-trees. This implies less chances of multiplexing traffic, thus limiting the improvement gained by performing grooming. In addition, the residual bandwidth of the wavelengths spanning a light-tree cannot be considered for any other demand. Both schemes incur in bandwidth wastage. Two types of architectures can support schemes mentioned: "opaques" and "transparents". However, these architectures 
do not allow the multiplexing of unicast and multicast traffic together, without incurring OEO conversions [2], [6], [7].

In this paper a novel architecture that allows the utilization of light-trees for grooming both unicast and multicast traffic without bandwidth wastage is proposed. The architecture allows to create a light-tree. Nevertheless, in the nodes where the optical signal is replicated, requests can be identified and eliminated from replicated signals that lead to undesired destinations. It also allows the node to inject traffic into the replicated signal reusing the eliminated bandwidth. Both operations are performed without OEO conversions. To the best of our knowledge, there have not been previous proposals of an architecture with these characteristics. A similar detection system was used in [8] and was named ORION (Overspill Routing in Optical Networks).

Additionally, in order to measure the benefits of the proposed architecture, a heuristic that minimizes the number of used wavelengths using the advantages of the architecture is proposed.

The rest of this paper is organized as follows. In Section II and Section III the problem and the proposed architecture are defined and explained respectively. In Section IV the heuristic is designed and detailed. Section V shows some simulation results evaluating the performance of the heuristic with the architecture. Finally some conclusions are presented in Section VI.

\section{Problem Definitions}

An example is used to explain the disadvantages of the classical methods used for routing unicast and multicast traffic. Let us consider a network of 4 nodes interconnected through optical links. Two sessions are considered. The first one $(S 1)$ being a unicast session $S 1:\{A\} \rightarrow\{C\}$, where the node $A$ is the source node and the node $C$ is the destination. The second one $(S 2)$ being a multicast session $S 2:\{A\} \rightarrow\{C, D\}$, where $C$ and $D$ are the destinations nodes. Routing these two sessions can be performed in the following ways:

- Light-TREes [2] (Figure 1(a)): sessions $S 1$ and $S 2$ are always routed through the same wavelengths. In this case, no OEO conversions are employed but traffic cannot be differentiated. As a consequence, all groomed traffic in a light-tree is routed to all the destinations. In the example, since the $S 1$ traffic should not be sunk at node $D$, there is bandwidth wastage. When a new request arrives $(S 3)$ are required to add new lightpath $(B \rightarrow D)$.

- LightPaths [1] (Figure 1(b)): two lightpaths are needed for routing both sessions $S 1$ and $S 2$. The first lightpath follows the path $A \rightarrow B \rightarrow C$ routing the sessions $S 1$ and $S 2$. The second lightpath routes session $S 2$ using the path $C \rightarrow B \rightarrow D$. It requires an additional wavelength, even though both demand could fit within one wavelength. In this case, there is also a waste of bandwidth, since spare bandwidth cannot be used. As in Light-tree, this scheme required lightpath additional to routed $S 3$.

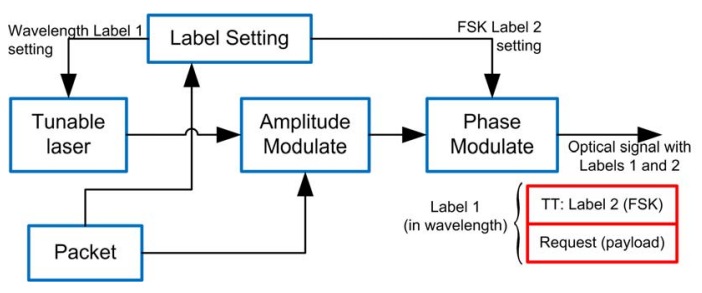

Fig. 2. S/G Light-tree Functionality

- Light-TRAILS [9] (Figure 1(c)): one light-trail is required for routing both sessions. A light-trail is an unidirectional optical bus. In the example, we can setup one between nodes $A$ and $D$ as $A \rightarrow B \rightarrow C \rightarrow B \rightarrow D$. Light-trails have the disadvantage that the path may contain repeated nodes and the length of a light-trail is limited. Please note that in our example we would need to consider one wavelength in $B \rightarrow C$ and another in $C \rightarrow B$.

- Link-BY-Link Routing [7] (Figure 1 $(d)$ ): this scheme routes traffic allowing OEO conversions on all nodes. Three lightpaths are used: $A \rightarrow B, B \rightarrow C$ and $B \rightarrow D$; one for each link. One lightpath routes sessions $S 1$ and $S 2$ together from node $A$ to node $B$. Node $B$ processes electronically the traffic and forwards session $S 1$ through the lightpath in $B \rightarrow C$ and $S 2$ through lightpaths $B \rightarrow C$ and $B \rightarrow D$. The wavelength bandwidth is well used, however it requires more electronic processing and OEO conversions.

In concrete, the problem arises when we have two (or more) sessions such that: $a$ ) both are originated in the same root, $b$ ) the wavelength capacity is enough for both sessions but, c) the destination nodes of one session is a subset of the other. In the previous example we consider one unicast and one multicast session, without lose of generality. As we appreciated in our example, there is no optical architecture based on WDM approach that can routes efficiently such a traffic: either residual bandwidth is wasted, or more OEO conversions are needed. While bandwidth plays an important role in the revenues of any ISP, the cost incurred by OEO conversion is the dominant cost in setting up the OTN. In general, the tendency is to setup a light-tree spanning to all possible destinations of a set of sessions, as shown in Figure $1(a)$.

Several studies tackle this problem. Reference [7] proposes an on-line technique called MulTicast Dynamic light-tree Grooming Algorithm (MTDGA). MTDGA is an algorithm that performs multicast traffic grooming with the objective of reducing the blocking probability by multiplexing unicast and multicast together. Reference [4] has also the objective of reducing the blocking probability, however it uses separate schemes for routing and grooming multicast and unicast traffic.

\section{Architecture Proposed}

We propose an architecture named Stop-and-Go Lighttree ( $S / G$ Light-tree). S/G Light-tree allows the grooming of 
unicast and multicast traffic together in a light-tree, hence reducing wastage bandwidth. S/G Light-tree take advantage of the circuit switching and packet switching technology. An S/G Light-tree allows a node to optically drop part of the multiplexed traffic in a wavelength without incurring on OEO conversions. Hence, once traffic is replicated, it prevents or stops the replicas from reaching undesirable destinations. Additionally, it enables a node to aggregate traffic in a passing wavelength also without incurring on OEO conversions. The optical processing capabilities required are available for typical rates of 2.5 or $10 \mathrm{~Gb} / \mathrm{s}$ [8].

The Figure 1(e) shows the solution of the previous example using an $\mathrm{S} / \mathrm{G}$ Light-tree. Session $S 1$ is dropped at node $B$ without the need of OEO conversions of the routed traffic in the wavelength. Session $S 3$ is added on the same wavelength of the S/G Light-tree at node $B$. While schemes $(d)$ and (e) efficiently use the bandwidth, scheme $(d)$ needs OEO conversions.

The S/G Light-tree architecture contains a "Switch architecture for multicasting with all-optical OXC" [2]. The Stopand-go functionality is held with the support of optical labels or "Traffic Tags" (TT) as we will shortly explain. Each packet in a wavelength contains a header carrying a TT field. Both unicast and multicast traffic can be marked with a TT. The length (in bits) of the TT field must be equal to the maximum number of destinations of any multicast session. The $i-$ bit in the TT field of a packet is used to indicate whether the packet should go to the $i$-destination of the S/G Light-tree. Hence, not all packets should be marked with a TT field, since there could be packets going to all destinations in the S/G Lighttree. TT can be inserted orthogonally to the packet data. The label information is FSK modulated on the phase, and the data is modulated on the amplitude of the carrier. Figure 2 shows in detail the procedure. The architecture has been designed for easy detection and processing of the TT.

Figure 3 shows the proposed node architecture. Initially, the optical fiber is demultiplexed in the wavelength channel (Demux). $\lambda_{2}$ carries the request $S 1$ and $S 2$ multiplexed electronically. $S 1$ is marked with a TT indicating that it should be stopped from going to $B . \lambda_{2}$ is switched (OSW1) in the Splitter and Amplifier Bank. In brief, the splitter replicates the incoming traffic to all the node's neighbors first, regardless of the TT field. Then, for each packet replica, the TT field is extracted in order to decide whether the packet should be stop from being forwarded to an undesired destinations.

For simplicity the figure shows only two detectors. A detection system consists of FSK Demod, 1x2 Fast Switch, Bit pattern Interpreter, Contention Resolution, Idle detection and delay lines. A small power is tapped from the wavelength and redirected to the FSK Demod, where the label gets demodulated and ready for interpretation. FSK Demod sends the TT field to the Bit pattern Interpreter. The TT-field is analyzed by an all-optical correlators in the Bit pattern Interpreter block.

If the interpreter-block identifies the TT field as that to be stopped, it communicates to its corresponding 1x2 Fast Switch in order to either drop or switched the packet towards the receiver $(\mathrm{Rx})$. A multiplexer is used to save receivers. These packets are later analyzed to decide whether they must be dropped (FREE), groomed in another S/G Light-tree or, dropped to the local network. A S/G Light-tree allows to add traffic to the wavelength as well, only when a free time slot is detected (Idle Detection). In our example, session $S 3$ can employ idle time slots (with a tunable lasers).

\section{HEURISTIC}

The multicast grooming strategy adopted in this paper has two main objectives: 1) to utilize the overall network resources as efficiently as possible using the S/G-tree architecture; and 2) minimize the maximum wavelength number. Figure 4 depicts the proposed heuristic: Grooming Algorithm for $S / G$ Light-tree (GA-S/G-trees). The inputs are:

- $N$ : number of nodes in the network.

- $W$ : maximum number of wavelength per fiber.

- $P_{m n}$ : physical topology, where $P_{m n}=P_{n m}=1$ indicates that there is one direct physical fiber links between nodes $m$ and $n$. If there is no fiber links between nodes $m$ and $n$, then $P_{m n}=0$.

- Every physical link between nodes $m$ and $n$ is associated with a weight $w_{m n}$. If $w_{m n}=0$ takes into account the number of hops.

- $C_{w}$ : capacity of each channel. For example, $C_{w}=O C-$ $192, O C-48$.

- $X$ set sessions, $|X|$ is number of sessions.

- $S_{i}$ : source nodes, $S_{i} \in X$.

- $D_{i}$ : set destination nodes for each session; $D_{i} \in X, D_{i}$ include unicast and multicast traffic.

- $B W_{i}$ : bandwidth request for each session.

- Every node is equipped with OEO converters, if it is necessary to use.

GA-S/G-trees sort all sessions from highest to lowest number of destinations including unicast and multicast request (Line 1). This will be used to increase the reuse of wavelengths and thus minimize the wavelength number.

The minimum-cost path heuristic (MCPH) [2] is employed to search a minimum-cost Steiner tree for a request $\left(X_{i}\right)$ in Line 3. Then, it eliminates $X_{i}$ from the session set $X$ if the request has a wavelength assigned (Line 6). GA-S/G-trees routes $X_{i}$ using this tree (named $Y$ ) and then search for session going to a subset of destinations of $Y$. In Line 8, to determine if $X_{j}$ can be groomed with the existing S/G Light-tree $Y$, it looks if the destination nodes in $X_{j}$ are contained in $Y$. The process continues until no demand can be groomed in $Y$.

\section{A. Complexity of the heuristic}

The computational complexity of GA-S/G-trees is given principally by the lines 7-17 in Figure 4 , directly related to MCPH. The time complexity of MCPH is $O\left(|X| \times N^{2} \log N\right)$, which guides the time complexity of GA-S/G-Trees to be $O\left(|X| \times N^{3} \log N\right)$. 


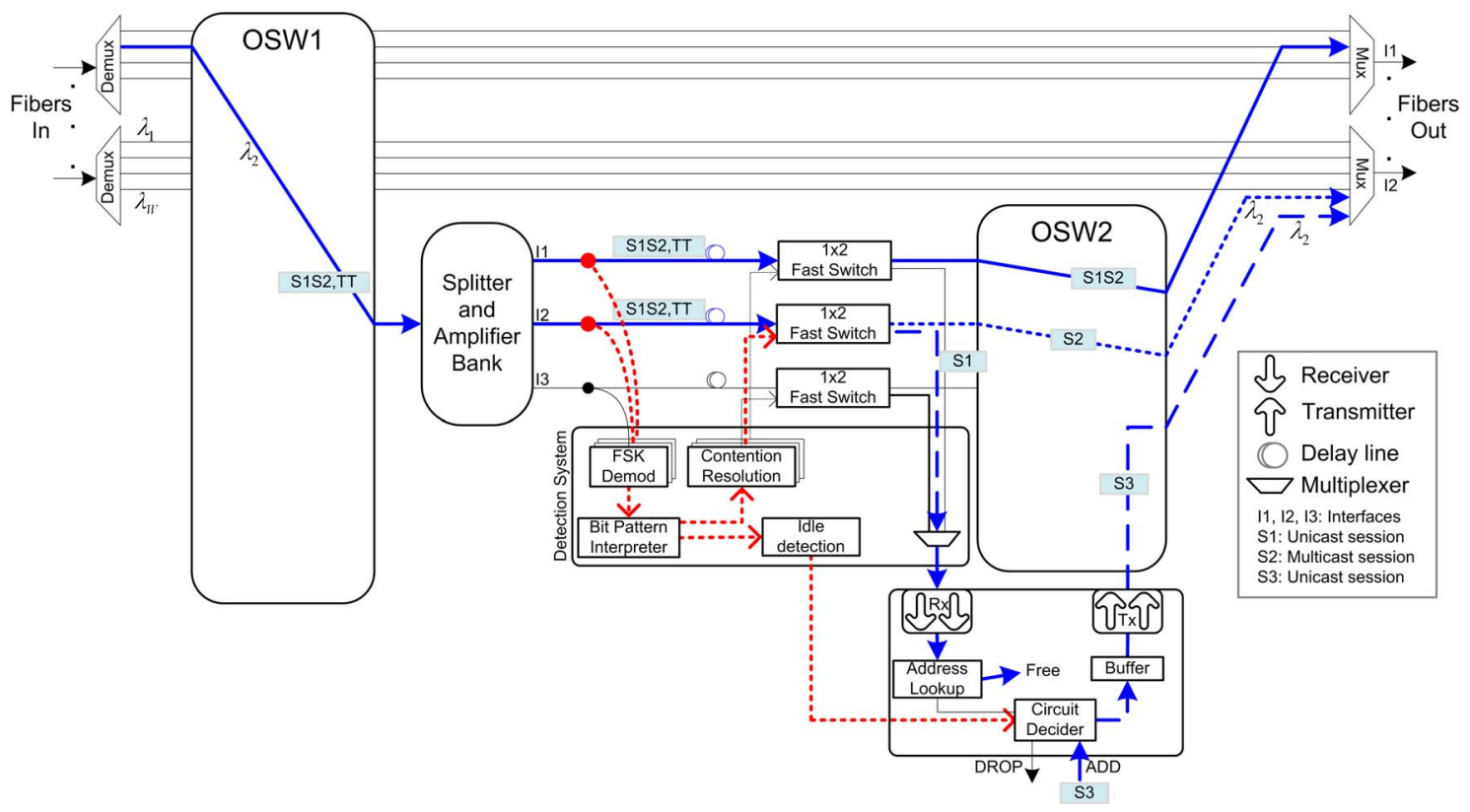

Fig. 3. Stop-and-Go Light-tree ( $S / G$ Light-tree)

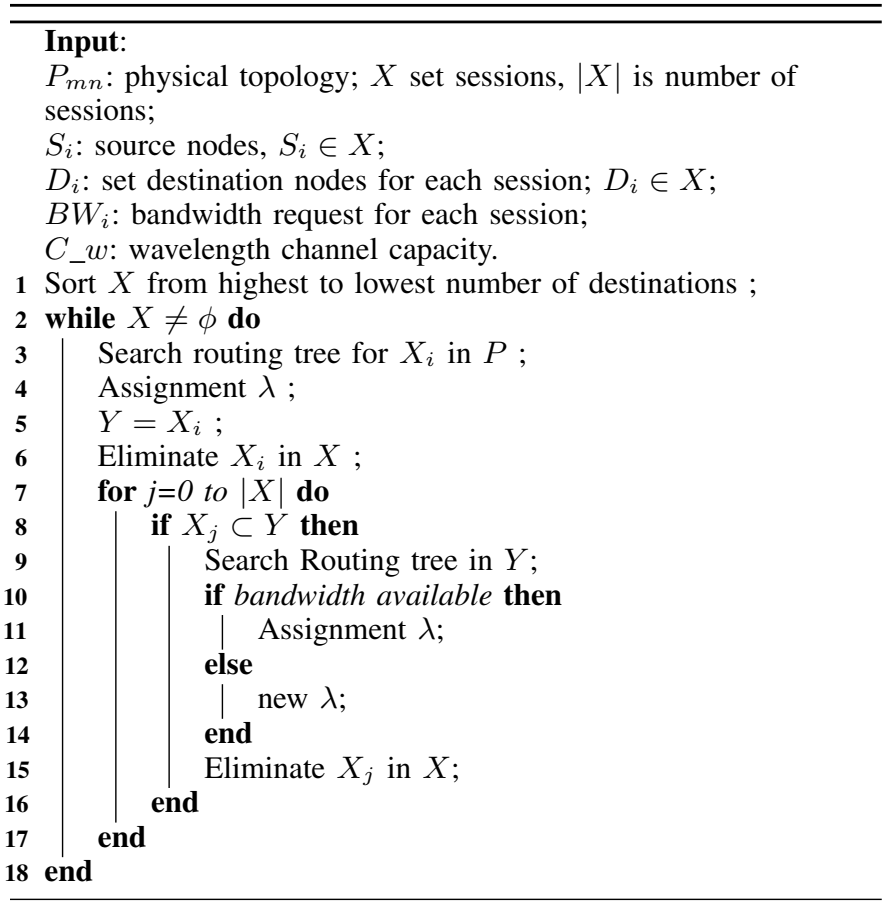

Fig. 4. Grooming Algorithm for S/G Light-tree (GA-S/G-trees)

\section{Performance Evaluation}

In this paper we compare our architecture with light-trees in term of $a$ ) used bandwidth, $b$ ) number of OEO conversions and, $c$ ) number of Splitter Bank employed. The comparison is made using the previously proposed algorithm in order to measure the efficiency of S/G Light-tree. Similarly, to measure the performance of the light-trees architecture the heuristic

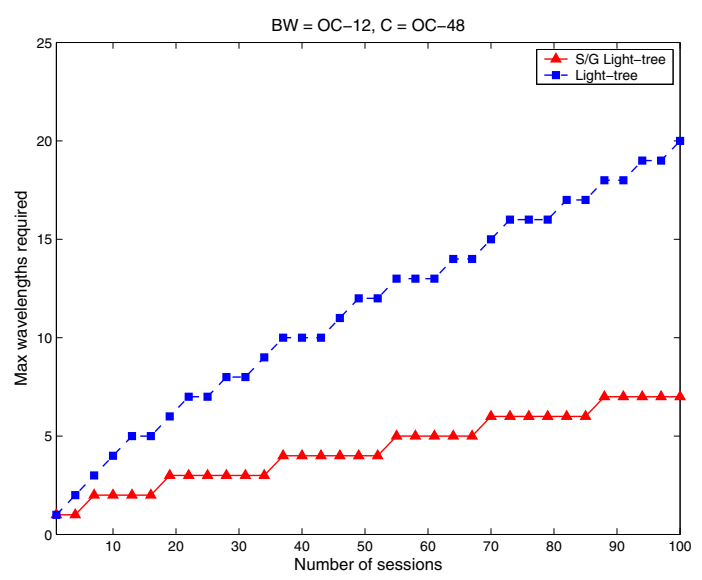

Fig. 5. Maximum wavelengths required for a path

proposed in [2] is used.

Simulations are performed using the NSFnet backbone network topology. The topology consists of 14 nodes with 21 bidirectional links.

Initially, sessions are generated randomly. For each run $40 \%$ of the sessions are unicast and $60 \%$ multicast. For each multicast session, the number of destinations is generated randomly as well. The capacity of a wavelength channel is set to $C_{w}=O C-48$ and $W=25$. All demands are fixed to $O C-12$.

For both algorithms performing multihop was allowed. The maximum number of wavelengths required for a path is presented on Figure 5. It can be appreciated that the number of required wavelengths for GA-S/G-trees is considerable minor than the ones required by a light-tree. In average GA-S/G-trees 


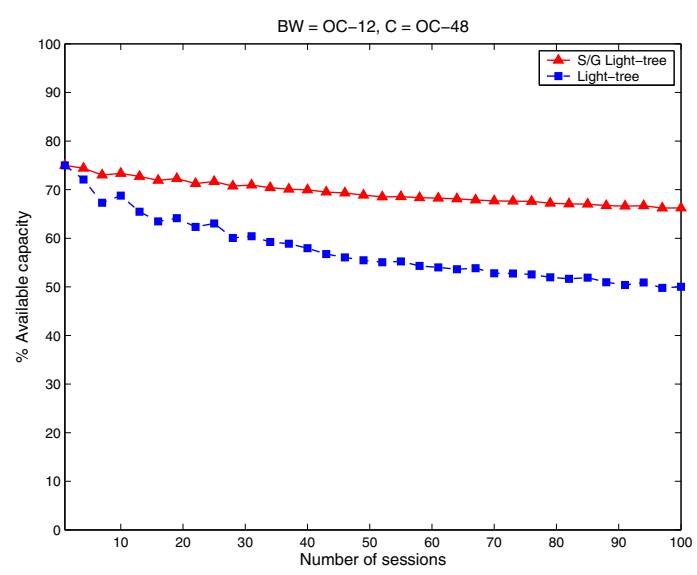

Fig. 6. Percentage available capacity in the network

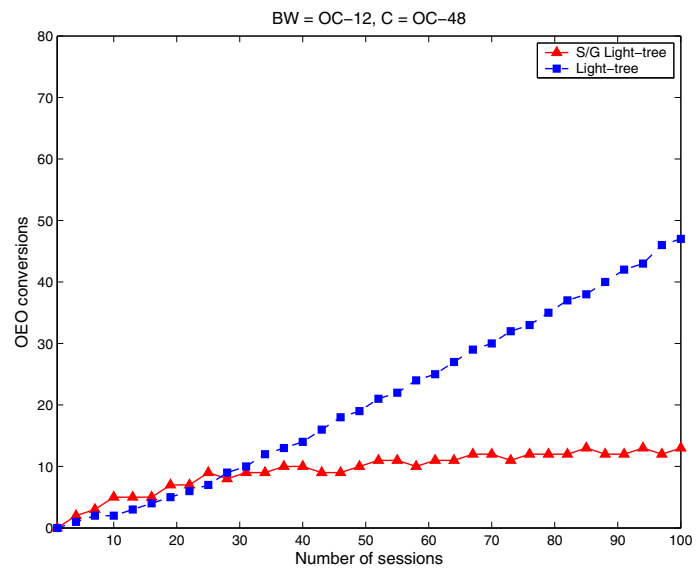

Fig. 7. Number OEO conversion in the network

use $40 \%$ wavelengths less than light-trees.

Figure 6 shows the available capacity percentage against the number of sessions. GA-S/G-trees presented and improvement of more than $20 \%$ regardless of the number of sessions.

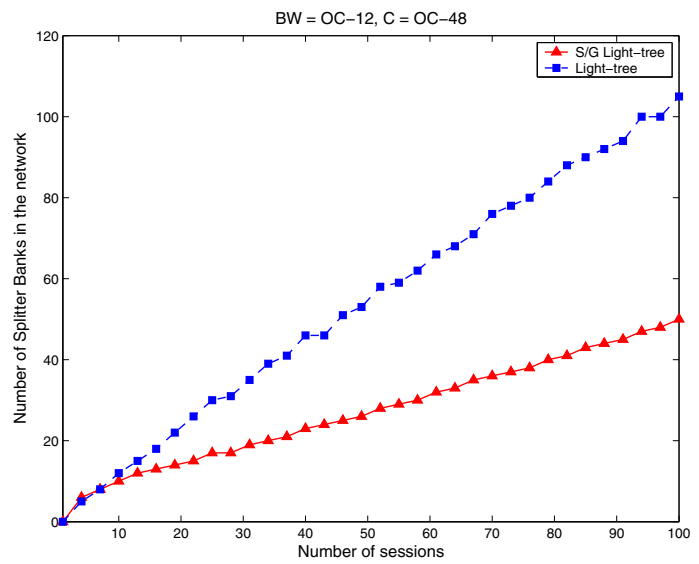

Fig. 8. Number Splitter Banks in the network
7 shows the number of OEO against the number of sessions. GA-S/G-trees presented a constant number of OEO conversions ( $\sim 11$ after 30 session), on the other hand light-trees presented an increasing number of OEO conversions (to 47 for 100 session) with the number of sessions.

The disadvantage of the proposed model resides in the costs of implementing the detection systems. Given that the system is composed by several FSK demod, 1x2 Fast Switch and one receiver; it could incur in a greater cost in the architecture. Figure 8 shows the minimum number of splitter banks required in the network. S/G Light-tree reduces the amount of splitter banks needed to route the sessions $(\sim 50 \%)$.

\section{CONCLUSIONS}

In this paper a novel architecture for performing multicast traffic grooming called S/G Light-tree has been described and evaluated. The architecture allows a node to drop part of the traffic optically multiplexed in a wavelength without incurring in OEO conversion. Additionally it enables a node to aggregate traffic in a passing wavelength also without incurring in OEO conversions.

For routing multicast and unicast traffic using S/G-tree taking advantage of its properties, an heuristic is proposed. The performance of the heuristic with GA-S/G-trees is simulated and evaluated. Simulation results show that the number of OEO conversions is considerably reduced and the network available capacity its improved.

\section{REFERENCES}

[1] K. Zhu and B. Mukherjee, "Traffic grooming in an optical wdm mesh network," Selected Areas in Communications, IEEE Journal on, vol. 20, no. 1, pp. 122-133, Jan. 2002.

[2] N. Singhal, L. Sahasrabuddhe, and B. Mukherjee, "Optimal multicasting of multiple light-trees of different bandwidth granularities in a wdm mesh network with sparse splitting capabilities," Networking, IEEE/ACM Transactions on, vol. 14, no. 5, pp. 1104-1117, Oct. 2006.

[3] I. Ferrel, A. Mettler, E. Miller, and R. Libeskind-Hadas, "Virtual topologies for multicasting with multiple originators in wdm networks," Networking, IEEE/ACM Transactions on, vol. 14, no. 1, pp. 183-190, Feb. 2006.

[4] A. Khalil, A. Hadjiantonis, C. Assi, A. Shami, G. Ellinas, and M. Ali, "Dynamic provisioning of low-speed unicast/multicast traffic demands in mesh-based wdm optical networks," Lightwave Technology, Journal of, vol. 24, no. 2, pp. 681-693, Feb. 2006.

[5] L. Liao, L. Li, and S. Wang, "Dynamic multicast traffic grooming in wdm mesh networks," in Next Generation Internet Design and Engineering, 2006. NGI '06. 2006 2nd Conference on, 3-5 April 2006, p. 5pp.

[6] L. Sahasrabuddhe and B. Mukherjee, "Light trees: optical multicasting for improved performance in wavelength routed networks," Communications Magazine, IEEE, vol. 37, no. 2, pp. 67-73, Feb. 1999

[7] X. Huang, F. Farahmand, and J. Jue, "Multicast traffic grooming in wavelength-routed wdm mesh networks using dynamically changing light-trees," Lightwave Technology, Journal of, vol. 23, no. 10, pp. 31783187, Oct. 2005.

[8] K. Vlachos, J. Zhang, J. Cheyns, Sulur, N. Chi, E. Van Breusegem, I. Monroy, J. Jennen, P. Holm-Nielsen, C. Peucheret, R. O'Dowd, P. Demeester, and A. Koonen, "An optical im/fsk coding technique for the implementation of a label-controlled arrayed waveguide packet router," Lightwave Technology, Journal of, vol. 21, no. 11, pp. 2617-2628, 2003.

[9] B. Wu and K. L. Yeung, "Light-trail assignment in wdm optical networks," in Global Telecommunications Conference, 2006. GLOBECOM 'O6. IEEE, Nov. 2006, pp. 1-5.

The number of OEO conversion is analyzed as well. Figure 